\title{
Aging of natural rubber studied via Fourier-transform rheology and double quantum NMR to correlate local chain dynamics with macroscopic mechanical response
}

\author{
Shouliang $\mathrm{Nie}^{\mathrm{a}}$, Jorge Lacayo-Pineda ${ }^{\mathrm{b}}$, Norbert Willenbacher ${ }^{\mathrm{c}}$, Manfred Wilhelm ${ }^{\mathrm{a}, *}$ \\ a Institute for Technical Chemistry and Polymer Chemistry, Karlsruhe Institute of Technology (KIT), Karlsruhe, 76131, Germany \\ ${ }^{\mathrm{b}}$ Research and Development, Continental Reifen Deutschland GmbH, Hannover, 30419, Germany \\ ${ }^{\mathrm{c}}$ Institute for Mechanical Process Engineering and Mechanics, Karlsruhe Institute of Technology (KIT), Karlsruhe, 76131, Germany
}

\section{H I G H L I G H T S}

- The development of rheological nonlinearity of NR during aging has been investigated.

- The evolution of the crosslink density and defects correlate well to rheological response.

- The parameter from FT-Rheology $I_{3 / 1}$ exhibits higher relative sensitivity.

- With the limitations of NMR and microscope, $I_{3 / 1}$ respond to the network change at both nano- and micrometer scales.

\begin{abstract}
A B S T R A C T
Nowadays mainly the linear rheological properties of natural rubber (NR) vulcanizate during aging are studied, even though NR is rheologically nonlinear under most application conditions. Here, the nonlinearity was quantified via Fourier transform rheology (FT-Rheology) using $I_{3 / 1}$, the relative intensity of the 3rd harmonic. The crosslink density and the content of defects were investigated by the ${ }^{1} \mathrm{H}$ double quantum nuclear magnetic resonance (DQ-NMR). For aerobic aging at $120^{\circ} \mathrm{C}$, the crosslink density increases, but later turns into a broader mesh size distribution with more defects. Accordingly, the rheological nonlinearity of the material increases in the earlier stage and then decreases. For mechanical aging, the nonlinearity continuously decreases as a function of the fatigue cycle numbers, whereas the local crosslink density of the polymer network remains constant. This behavior can be assigned to the micro-cracks generated along adjacent network defects, which are larger than the detection scale of DQ-NMR. Compared to the linear rheological parameters, storage modulus $G^{\prime}$ and loss factor $\tan \delta, I_{3 / 1}$ exhibits higher sensitivity to the aging of crosslinked NR under both high temperature aerobic and mechanical fatigue conditions.
\end{abstract}

\section{Introduction}

The demand of natural rubber (NR) has been growing intensively after the discovery of the vulcanization process, i.e. the crosslinking of NR by sulfur with heat-treatment [1,2]. The worldwide consumption of NR in 2018 reached 13.9 million tonnes [3], which is almost the total consumption of all types of synthetic rubbers. The excellent elasticity and good durability [4] of crosslinked NR make it irreplaceable in rubber industry, with products ranging from tires to hoses. Those rubber products are used mostly over long term under different environmental or mechanical fatigue conditions. Crosslinked NR and its composites can be either chemically aged [4,5] by such as heat, light, oxygen, or physically aged $[4,6]$ under large deformations. Therefore, the study of the NR aging process and the development of the characterization method are of great importance with a view on both fundamental and practical aspects to evaluate the longevity and safety of the rubber products.

The exposure of NR vulcanizate in air at elevated temperatures will lead to structural changes of the sulfur linkages, as well as molecular chain scission reactions [5,7-11]. At extended time of heating, it is suggested [5] that new crosslinks are formed and polysulfidic crosslinks are shortened into e.g. mono- or disulfide. In the presence of oxygen,

\footnotetext{
* Corresponding author.

E-mail address: manfred.wilhelm@kit.edu (M. Wilhelm).
} 
both the sulfidic crosslinks and the unsaturated polymer chain may undergo a scission reaction, accompanied by the formation of some polar groups $[7,11]$. Consequently, the mechanical properties of the products deteriorate with e.g. the increase of loss factor $\tan \delta$ and the decrease of the elongation at break, due to the change of crosslinks types, crosslinking mesh size and mesh size distribution. In addition, NR products such as tires, air springs and other components are designed to endure mechanical fatigue under long term large deformation during their service life. The network flaws initially existing in the vulcanizate would grow into small cracks $[6,12]$, resulting in a deterioration of mechanical properties, and finally, in failure.

To study the aging of crosslinked NR products, it is both essential and practical to evaluate their mechanical properties during aging. It is also well known that crosslinked rubber and its composites typically exhibit nonlinear viscoelasticity under large strain $[13,14]$. Oscillatory deformation measurements are commonly employed to evaluate their dynamic mechanical properties, e.g. the storage and loss moduli, $G^{\prime}\left(\omega_{1}\right.$, $\left.\gamma_{0}\right), G^{\prime \prime}\left(\omega_{1}, \gamma_{0}\right)$ as a function of shear strain amplitude, $\gamma_{0}$, at an excitation frequency of $\omega_{1} / 2 \pi$. However, this is not adequate in the nonlinear viscoelastic regime, where the stress response wave has already been distorted and the $G^{\prime}$ and $G^{\prime \prime}$ only give access to the linear portion of it. Substantial information captured in the stress signal is lost. Therefore, it is advantageous to quantify the nonlinear portion of the wave, i.e. the rheological nonlinearity, to fully understand the physical meaning of the nonlinear response. Fourier-transform rheology (FTRheology) as a recently developed technique by Wilhelm et al. [15-17] can quantify the nonlinearity by converting the stress response wave from the time domain to frequency domain. Parameters such as the relative intensity of the 3rd harmonic, $I_{3 / 1}\left(\gamma_{0}\right)$, can be used as the simplest way to measure rheological nonlinearity at specified oscillatory deformation frequency and strain amplitude. Thereafter, FTRheology has been applied to study rheological nonlinearity of various soft materials, including polymer melts [18], polymer blends [19] and rubber compounds in unvulcanised state [20,21], but not yet crosslinked rubber and its aged products.

To determine the change of crosslink density and structures of the NR networks during aging, several approaches have been developed such as equilibrium swelling in solvents [22-24], freezing-point depression [25,26], Fourier transform infrared spectrometry (FTIR) [27] and high resolution nuclear magnetic resonance spectrometry (NMR) $[11,28]$. More recently, the ${ }^{1} \mathrm{H}$ double quantum NMR (DQ-NMR) technique established by Saalwächter et al. [29,30] can precisely quantify the average network density, mesh size distribution and network defects of elastomers based on the local polymer chain dynamics. Superior to equilibrium swelling method, the ${ }^{1} \mathrm{H}$ DQ-NMR measurement can be conveniently performed on a low-field time domain NMR in around $2 \mathrm{~h}$ without solvent treatment of the sample. In addition, compared to the high-field NMR, the smaller size low-field setup is less interfered by the magnetic susceptibility if conductive fillers such as carbon black (CB) were blended into the rubbers $[31,32]$.

In present work, crosslinked NR with and without CB were either aged chemically by hot air, or physically by mechanical fatigue at different time intervals. For the first time, the development of rheological nonlinearity of NR vulcanizates during aging has been studied. The relative sensitivity of the nonlinear rheological parameter from FTRheology, $I_{3 / 1}$, to the effect of aging has been compared with that of the linear rheological parameters, $G^{\prime}$ and $\tan \delta$. The evolution of the crosslink density and the content of network defects during aging has been revealed and compared, relying on the ${ }^{1} \mathrm{H}$ DQ-NMR technique.

\section{Experimental}

\subsection{Mixing and vulcanization}

All the compounds used in this work were prepared by Continental Reifen Deutschland GmbH, Hannover, Germany. The formulations of
Table 1

Formulations of the NR compounds.

\begin{tabular}{lrrrr}
\hline Ingredients & Sample: NR1 & NRCB1 & NRCB0 & NRCB2 \\
\cline { 2 - 5 } & $\mathrm{phr}$ & $\mathrm{phr}$ & $\mathrm{phr}$ & $\mathrm{phr}$ \\
\hline Natrual rubber (NR) & 100.0 & 100.0 & 100.0 & 100.0 \\
Carbon black N339 (CB) & 0 & 50.0 & 50.0 & 50.0 \\
Zinc oxide & 3.0 & 3.0 & 3.0 & 3.0 \\
Stearic acid & 1.0 & 1.0 & 1.0 & 1.0 \\
6PPD $^{\mathrm{a}}$ & 1.8 & 1.8 & 0 & 3.6 \\
CBS $^{\mathrm{b}}$ & 1.1 & 1.1 & 1.1 & 1.1 \\
Sulfur & 1.1 & 1.1 & 1.1 & 1.1 \\
\hline
\end{tabular}

a $\mathrm{N}$-(1, 3-dimethylbutyl)- $\mathrm{N}^{\prime}$ - phenyl-p-phenylenediamine.

b $\mathrm{N}$-cyclohexyl-2-benzothiazole sulfenamide

the compounds are shown in Table 1. Crosslinked NR without filler or with CB N339 were prepared, namely NR1 and NRCB1, with 1 here denoted as the normal dosage of the anti-oxidant to simulate the tire tread compounds. The loading of N339 was $50 \mathrm{phr}$ (parts per hundred rubber by weight), with reference to ASTM-D3192 [33] for CB evaluation in NR as well as common practice in rubber industry. Two extra CB filled samples with zero or double dosage of the anti-oxidant were prepared, which were named as NRCB0 and NRCB2, accordingly. Other ingredients were identical with each other. All compounds were mixed in a $2 \mathrm{~L}$ internal mixer (Harburg-Freudenberger, Hamburg, Germany) in a two-step mixing process. In the first step, all ingredients except the vulcanization system were mixed for $6 \mathrm{~min}$ at a temperature of approximately $150^{\circ} \mathrm{C}$ with a rotor speed of 30-40 rpm (revolutions per minute). In the second step, the vulcanization system was added and the compound was finalized by mixing for $2.5 \mathrm{~min}$ at $100^{\circ} \mathrm{C}$ with a rotor speed of $30 \mathrm{rpm}$. All compounds were vulcanized by pressing them into $1.5 \mathrm{~mm}$ thick flat sheets at $160^{\circ} \mathrm{C}$ within optimum curing time (the time at the maximum torque in the rheo-curves from the curemeter).

\subsection{Aging of crosslinked rubber}

The $1.5 \mathrm{~mm}$ thick vulcanized rubber sheets were aged either statically at elevated temperatures in the presence of air (hot air aging) or mechanically fatigued (mechanical aging) by cyclic stretching at room temperature. For hot air aging, the rubber sheets were hung in oven at 70 or $120^{\circ} \mathrm{C}$ in air atmosphere for varying days. For mechanical aging, the rubber sheets of NRCB1 were cut into dumbbell shape specimen with a working area of $4 \times 25 \mathrm{~mm}^{2}$. The dumbbell specimens were then mounted on the fatigue machine (Fatigue to Failure Tester, Alpha Technologies, Hudson, USA), with reference to ASTM-D4482 [34]. The specimens were fatigued under cyclic stretching from the strain amplitude $\varepsilon=0-90 \%$ at a frequency of $1.7 \mathrm{~Hz}$ (100 cycles per minute) and varying kilocycle numbers, but removed from the machine before failure, i.e. before cracks on the specimens were visually observable or the modulus dropped more than $50 \%$.

\subsection{Rheology}

Both original and aged $1.5 \mathrm{~mm}$ thick rubber specimens were cut into $3 \mathrm{~mm}$ wide strips and then fixed on ARES-G2 strain-controlled rheometer (TA Instruments, New Castle, USA) with a rectangle fixture at a gap distance of $2 \mathrm{~mm}$. For the dumbbell shape specimens after mechanical aging, only their working areas were selected for measurements. The specimens were investigated via strain sweep from $\gamma_{o}=0.01-200 \%$ at an oscillatory frequency of $\omega_{1} / 2 \pi=1 \mathrm{~Hz}$ and room temperature to obtain both linear rheological results and the FTRheology parameter, $I_{3 / 1}$, as a function of $\gamma_{0}$. Twenty data points per decade of the strain amplitude were measured with ten oscillation cycles for the acquisition of each data point. At least three specimens were tested for each sample to evaluate the reproducibility. 


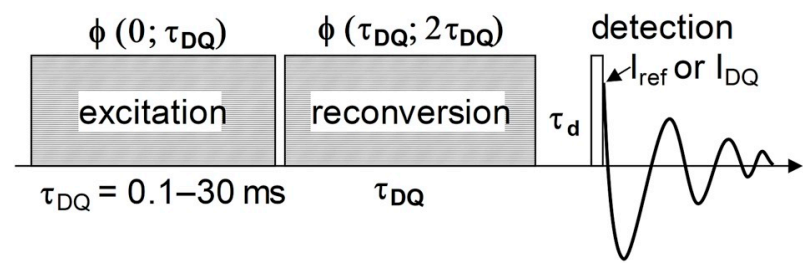

Fig. 1. Scheme of the DQ-NMR measurement with Baum-Pines sequence $[35,36]$ for dipolar coupling excitation and reconversion.

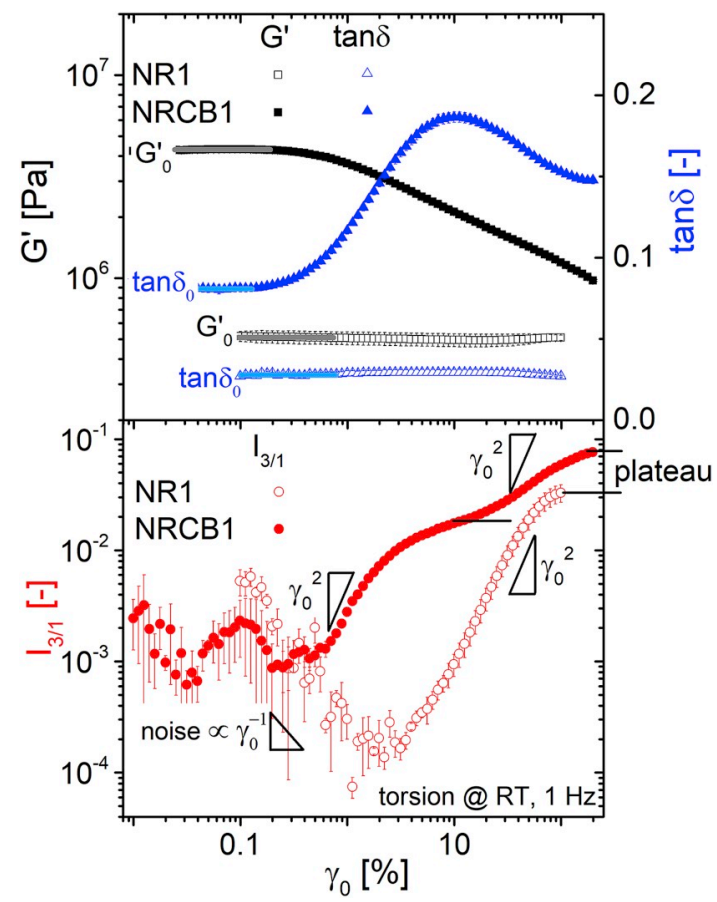

Fig. 2. Strain dependence of the linear and nonlinear rheological parameters of crosslinked natural rubber, with and without carbon black, on ARES-G2 under oscillatory torsion at an excitation frequency of $\omega_{1} / 2 \pi=1 \mathrm{~Hz}$ and room temperature (RT). The data are the average value obtained from three specimens of each sample with the standard deviation as the error bars on the symbols.

\subsection{Double quantum NMR}

Proton double quantum NMR ( ${ }^{1} \mathrm{H}$ DQ-NMR) is a versatile and robust technique to characterize the structure of crosslinked rubber materials $[29,30]$ by measuring the polymer chain dynamics. The content of network defects, the network density and its distribution can be quantitatively described.

The $1.5 \mathrm{~mm}$ thick rubber sheets were cut into $8 \mathrm{~mm}$ diameter disks and piled into $\mathrm{OD}=10 \mathrm{~mm}$ NMR glass tubes with a pile height lower than $8 \mathrm{~mm}$. For the dumbbell shape samples after mechanical aging, only the working areas were selected and cut into roughly $2 \times 2 \times 1.5 \mathrm{~mm}^{3}$ cubes before filling into the NMR glass tubes as above. DQ-NMR measurement were conducted on a $20 \mathrm{MHz}$ NMR (Minispec mq20 NMR Analyzer, Bruker, Rheinstetten, Germany) by applying a Baum-Pines NMR pulse sequence $[35,36]$ as the scheme shown in Fig. 1. The measurement temperature was $60^{\circ} \mathrm{C}$, which was more than $T_{g}+100{ }^{\circ} \mathrm{C}$ of NR (onset $T_{g}$ of NR was $-67^{\circ} \mathrm{C}$ [37]) as the prerequisite to measure the residual dipolar coupling constant at the entanglement level [29]. The $90^{\circ}$ and $180^{\circ}{ }^{1} \mathrm{H}$ pulse lengths of the crosslinked NR were 2.7 and $4.9 \mu$ s, respectively. The conditioned samples at the measurement temperature were exited and reconverted at different evolution time intervals, $\tau_{D Q}$, from 0.08 to $30 \mathrm{~ms}$. The corresponding reference intensity and double quantum build-up intensity, $I_{r e f}$ and $I_{D Q}$, were acquired separately. Each sample was

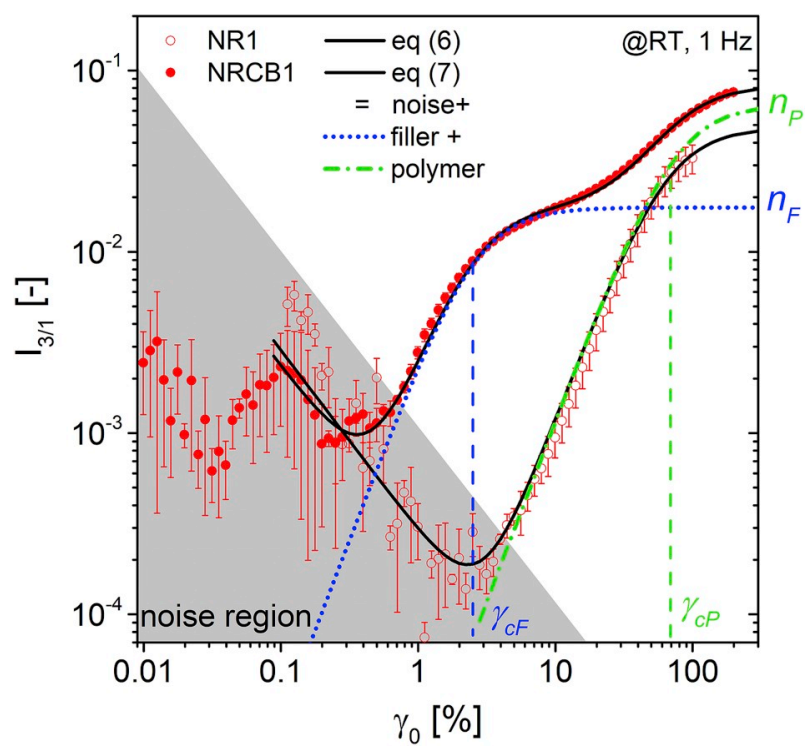

Fig. 3. Comparison of the critical strain amplitudes for $I_{3 / 1}\left(\gamma_{0}\right)$ and corresponding nonlinearity of the unfilled and filled NR vulcanizate by fitting the data with equation (6) and equation (7), respectively, as the black solid lines. The contribution of the noise term as equation (5) has been included.

Table 2

FT-Rheology fitting parameters from equations (6) and (7) for crosslinked NR networks without and with CB fillers.

\begin{tabular}{lllll}
\hline Sample & Filler network & \multicolumn{3}{l}{ Polymer network } \\
\cline { 2 - 5 } & $\mathrm{n}_{\mathrm{F}}$ & $\gamma_{\mathrm{CF}}$ & $\mathrm{n}_{\mathrm{P}}$ & $\gamma_{\mathrm{CP}}$ \\
\hline NR1 & - & - & 0.049 & $64 \%$ \\
NRCB1 & 0.0176 & $2.6 \%$ & 0.065 & $75 \%$ \\
\hline
\end{tabular}

measured three times to evaluate the reproducibility of the data. The $I_{r e f}\left(\tau_{D Q}\right)$ and $I_{D Q}\left(\tau_{D Q}\right)$ data were analyzed following the procedure described in reference [38].

\subsection{Light microscope}

Both the unaged and aged samples were cut with a sharp scalpel and the cross-section surfaces were observed under a light reflection microscope (VHX 900F, Keyence Corporation, Osaka, Japan) at a magnification of $1000 \times$. For mechanically fatigued samples in dumbbell shape, the cross-section was selected only from the middle working area of the specimens.

\section{Results and discussion}

\subsection{FT-Rheology of NR vulcanizate}

The strain sweep results of the crosslinked natural rubbers before aging, both the unfilled and the CB filled (NR1 and NRCB1) are compared in Fig. 2. The storage modulus $G^{\prime}$ of NRCB1 in linear regime was 10 times higher than that of NR1 due to the reinforcement of CB. The $G^{\prime}$ of NRCB1 also exhibited more obvious strain dependence with a stronger decrease at high strain amplitudes, i.e. Payne effect [39], resulting from the filler. The mechanical loss factor $\tan \delta$ was also increased by the filling of $\mathrm{CB}$, and developed a maximum at $\gamma_{o}=10 \%$, which may be attributed to the deagglomeration of the filler networks under strain as suggested by literature $[14,40,41]$. The storage modulus and $\tan \delta$ within the linear viscoelastic regime $\left(G^{\prime}{ }_{0}\right.$ and $\left.\tan \delta_{O}\right)$ as indicated by the solid lines in Fig. 2, were going to be used as the linear rheological parameters for the following study of rubber aging. 

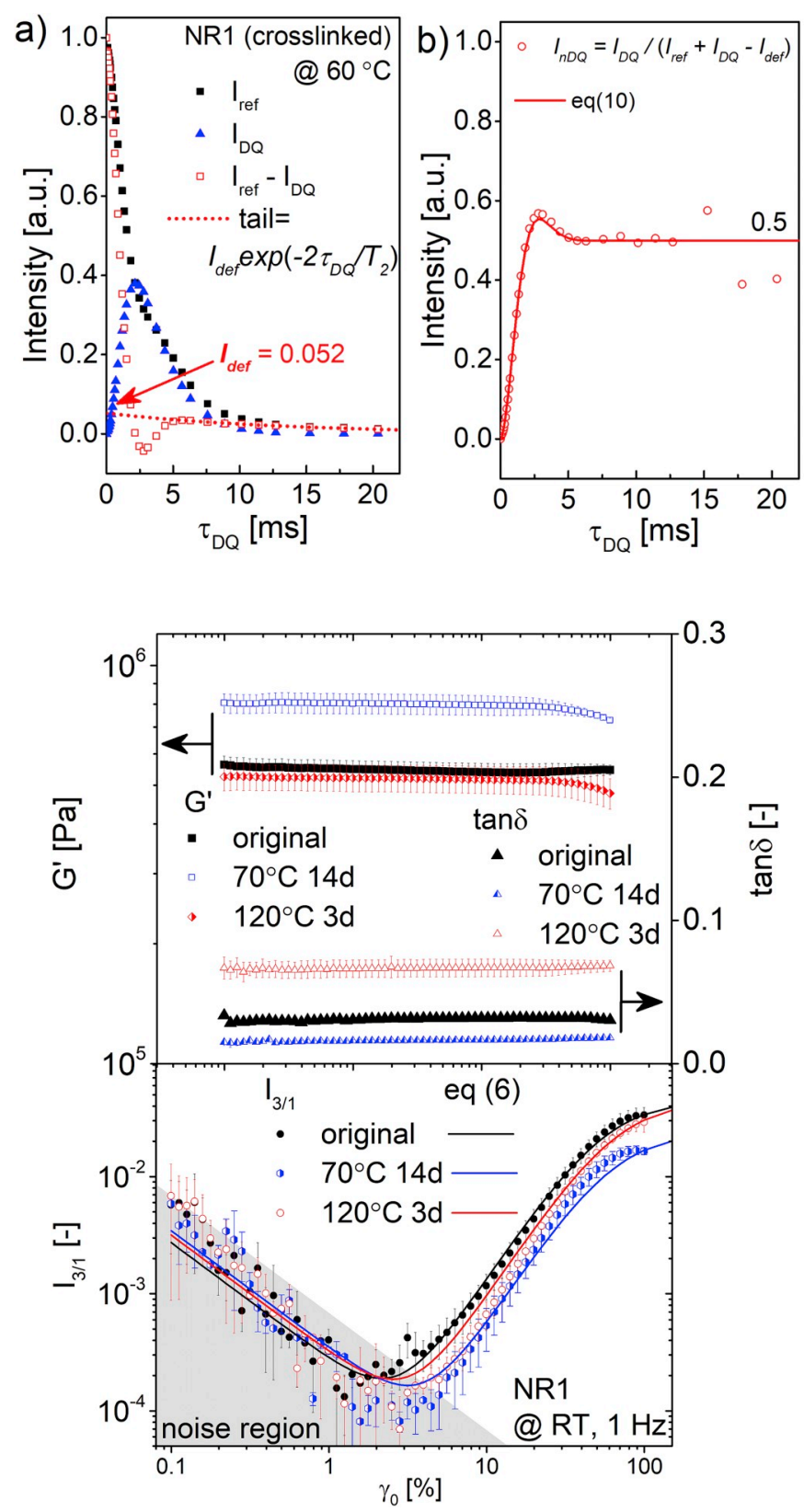

Fig. 5. Linear and nonlinear rheological properties of crosslinked NR without fillers before and after hot air aging at two typical conditions, $70^{\circ} \mathrm{C}$ for 14 days and $120^{\circ} \mathrm{C}$ for 3 days.

The quantification of rheological nonlinearity via FT-Rheology can be elucidated with a material under a sinusoidal shear. In Euler's form, the deformation [18] is

$\gamma(\mathrm{t})=\gamma_{0} \exp \left[\mathrm{i}\left(\omega_{1} \mathrm{t}\right)\right]$

The stress response in linear viscoelastic regime of the material can be described as a function of the modulus,

$\sigma(t)=\mathrm{G}_{0} \gamma_{0} \exp \left[\mathrm{i}\left(\omega_{1} \mathrm{t}+\delta\right)\right]$,

with $G_{O}$ as the shear modulus at the excitation frequency and $\delta$ as the phase lag angle.

In the nonlinear viscoelastic regime, the periodic stress response is not sinusoidal anymore but still symmetric. The modulus $G_{0}$ in equation (2) can be described by Taylor expansion series with even exponents, so that

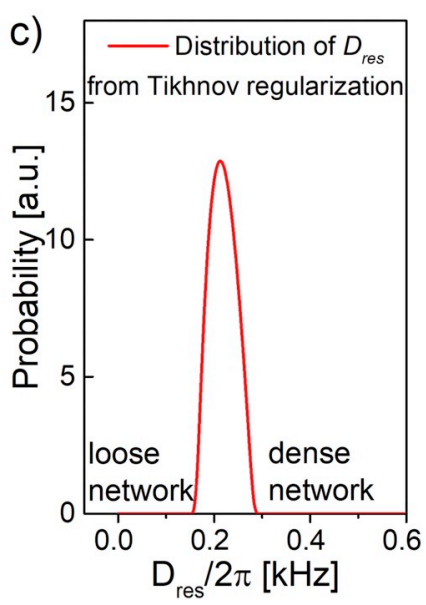

Fig. 4. The treatment procedure of DQNMR data using the crosslinked NR as an example: a) the double-quantum build-up, $I_{D Q}$, and the reference decay, $I_{r e f}$, as a function of the double quantum evolution time $\tau_{D Q}$ were obtained from the solid state NMR measurement at $60^{\circ} \mathrm{C}$. The deference $I_{r e f}-I_{D Q}$, can be used to detect the content of network defect, $I_{\text {def }}$ (the dashed line). b) Double quantum intensity was normalized into $I_{n D Q}$ and submitted to Tikhonov regularization algorithm [38]. Finally c) the distribution of residual dipolar coupling constant, $D_{\text {res }}$, representing the crosslink density, was obtained.

$$
\begin{aligned}
& \sigma(t)=\left[G_{1}+G_{3} \gamma^{2}(t)+G_{5} \gamma^{4}(t)+\ldots\right] \gamma_{0} \exp \left[i\left(\omega_{1} t+\delta\right)\right] \\
& =G_{1} \gamma_{0} \exp \left[i\left(\omega_{1} t+\delta\right)\right]+G_{3} \gamma_{0}^{3} \exp \left[i\left(3 \omega_{1} t+\delta_{3}\right)\right]+G_{5} \gamma_{0}{ }^{5} \exp \left[i \left(5 \omega_{1} t\right.\right. \\
& \left.\left.+\delta_{5}\right)\right]+\ldots \\
& =I_{1} \exp \left[i\left(\omega_{1} t+\delta\right)\right]+I_{3} \exp \left[i\left(3 \omega_{1} t+\delta_{3}\right)\right]+I_{5} \exp \left[i\left(5 \omega_{1} t+\delta_{5}\right)\right]+\ldots,
\end{aligned}
$$

where $G_{1}, G_{3}, G_{5} \ldots$ and $\delta, \delta_{3}, \delta_{5} \ldots$ are the prefactors and the phase angles, respectively, at the excitation frequency, the 3rd higher harmonic, the 5th higher harmonics and so on. The intensities in the FTRheological spectrum are denoted as $I_{1}, I_{3}, I_{5} \ldots$, accordingly. The rheological nonlinearity can be quantified by the relative intensity of the 3rd harmonic,

$$
\mathrm{I}_{3 / 1} \equiv \mathrm{I}_{3} / \mathrm{I}_{1}=\left(\mathrm{G}_{3} \gamma_{0}^{3}\right) /\left(\mathrm{G}_{1} \gamma_{0}\right)
$$

At small strain amplitudes, $I_{3 / 1}\left(\gamma_{0}\right)$ of both NR1 and NRCB1 was dominated by noise with a slope of -1 in the double logarithmic scale as indicated in Fig. 2 (the lower graph), a typical phenomenon observed in FT-Rheology [16-21]. In this region, the small torque response from the measurement is beyond the resolution of the torque transducer. Therefore, $I_{3}$ is dominated by the absolute noise from the transducer which the value should be constant for the identical rheometer at the same torque level of the transducer. Therefore,

$I_{3 / 1}\left(\right.$ at small $\left.\gamma_{0}\right)=I_{3} / I_{1} \propto \frac{\text { constant noise }}{G_{1} \gamma_{0}}=L_{\text {noise }} / \gamma_{0} \propto \gamma_{0}^{-1}$,

with the constant $L_{\text {noise }}$ stands for the level of the noise during the measurement.

In the $I_{3 / 1}\left(\gamma_{0}\right)$ of NRCB1, there is a peak around $\gamma_{0}=0.1 \%$. Similar peaks are also observed from polymer melts [18] and unvulcanized rubbers [21]. However, the origin is still not clear. In present work, those peaks are out of our focus and thus not taken for discussion.

After the noise region, $I_{3 / 1}$ starts to monotonically increase at $\gamma_{o}=3 \%$ and $\gamma_{o}=0.3 \%$ for NR1 and NRCB1, respectively. For NR1, the $I_{3 / 1}\left(\gamma_{0}\right)$ increases quadratically as $I_{3 / 1}=\left(G_{3} \gamma_{0}{ }^{3}\right) /\left(G_{1} \gamma_{0}\right)$, where $G_{3} / G_{1}$ are considered as constant in this region. At higher strain amplitude, the increase of $I_{3 / 1}\left(\gamma_{0}\right)$ levels off and reaches a plateau after $\gamma_{0}=100 \%$. The growth shape of $I_{3 / 1}\left(\gamma_{0}\right)$, i.e. quadratic increase and finally reaching a plateau, has also been observed from other viscoelastic materials with both experimental [16,18] and modelling [42] approaches. Considering a quadratic growth as a function of $\gamma_{0}$ and also the presence of a plateau at infinite $\gamma_{0}$, the $I_{3 / 1}\left(\gamma_{0}\right)$ can be empirically described via sigmoidal functions with squared exponent. The following equation form performs better fitting on the experimental data (see supplementary information): 

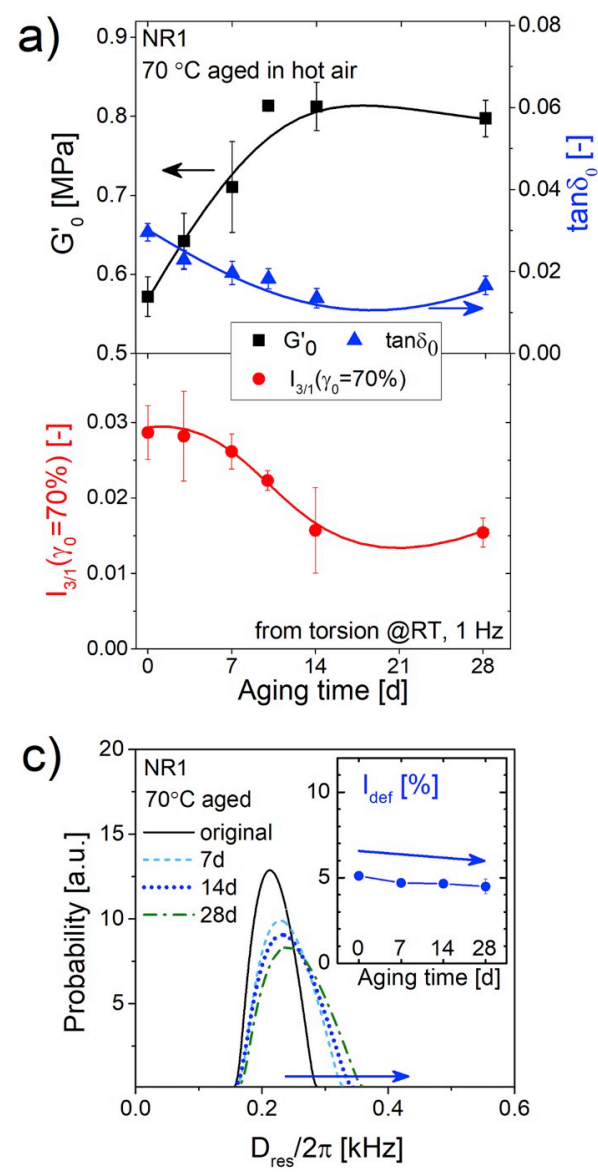

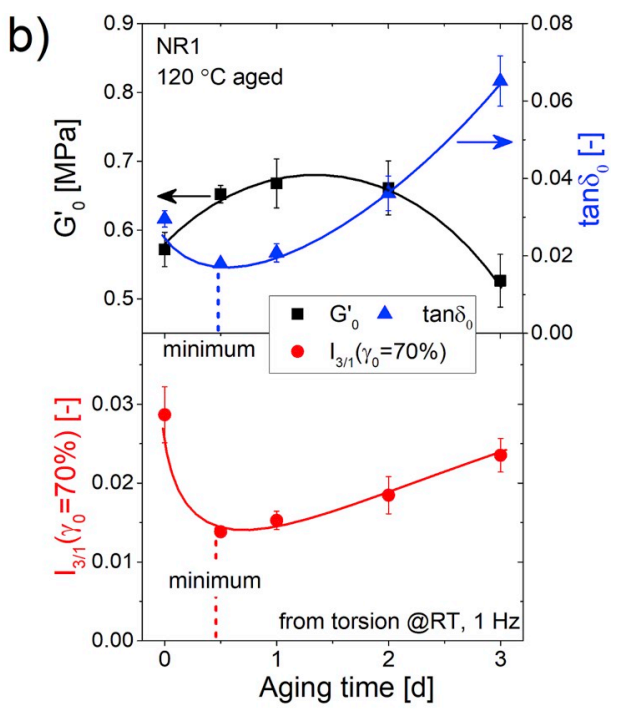

d)

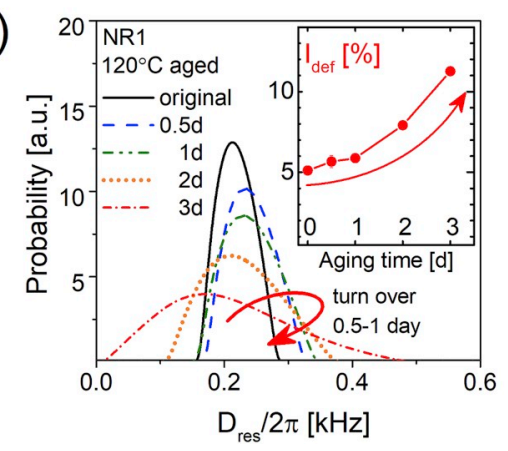

Fig. 6. The development of the elasticity and loss factor in linear viscoelastic regime, $G^{\prime}{ }_{0}$ and $\tan \delta_{0}$, as well as the nonlinearity at critical strain amplitude, $I_{3 / 1}\left(\gamma_{0}=70 \%\right)$, of NR vulcanizate during aerobic aging at a) $70{ }^{\circ} \mathrm{C}$ and b) $120^{\circ} \mathrm{C}$, respectively. The evolution of the crosslink density distribution and network defects (the insets) at two aging temperatures are represented in c) and d) correspondingly. The rheological parameters were obtained from oscillatory torsion at room temperature and $1 \mathrm{~Hz}$. The lines and arrows were drawn to guide the eye. The distribution of residual dipolar coupling constant, $D_{\text {res}}$, and content of network defects, $I_{d e f}$, were acquired from ${ }^{1} \mathrm{H}$ DQ-NMR measurements at $60^{\circ} \mathrm{C}$.
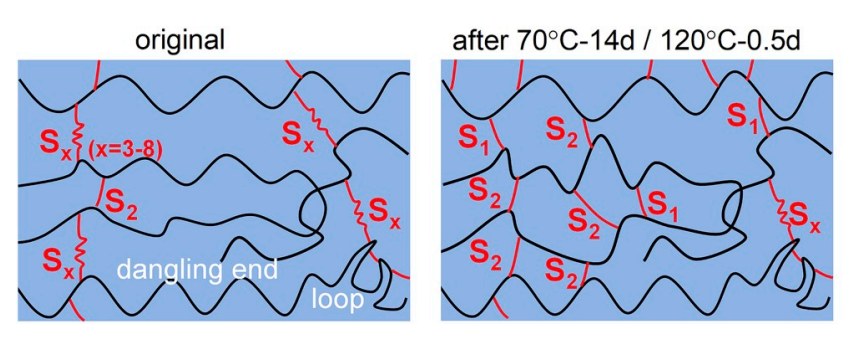

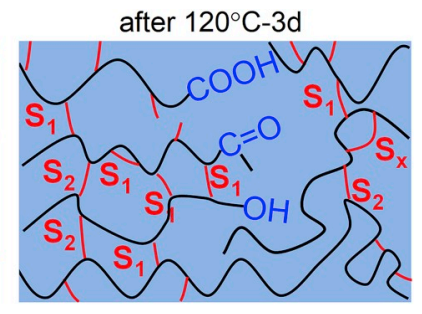

Fig. 7. Schematic diagrams of the evolution of vulcanized NR network structures under progressive aging in air at elevated temperatures.

$$
I_{3 / 1}\left(\gamma_{0}\right)=\frac{L_{\text {noise }}}{\gamma_{0}}+n_{P} * \frac{\left(\frac{\gamma_{0}}{\gamma_{c P}}\right)^{2}}{1+\left(\frac{\gamma_{0}}{\gamma_{C P}}\right)^{2}},
$$

where $\gamma_{c}$ is the critical strain amplitude before which $I_{3 / 1}\left(\gamma_{0}\right)$ grows quadratically and afterwards levels off. The pre-factor, $n_{P}$, is the limit of $I_{3 / 1}\left(\gamma_{0}\right)$ at infinite high strain amplitude, i.e. the utmost nonlinearity in terms of $I_{3 / 1}\left(\gamma_{0}\right)$, with the index "P" stands for the polymer network. At the critical strain amplitude $\gamma_{c}, I_{3 / 1}\left(\gamma_{c}\right)=n / 2$, indicating the network would reach half of its utmost nonlinearity.

Compared to the unfilled sample, the $I_{3 / 1}\left(\gamma_{0}\right)$ of NRCB1 notably increases at the medium strain amplitude near $\gamma_{o}=10 \%$. The increase of rheological nonlinearity at medium strain amplitude was suggested to be the contribution of the filler network in the composite, as discussed in previous work [21]. Furthermore, at both before and after $\gamma_{o}=10 \%$, the growth of $I_{3 / 1}\left(\gamma_{0}\right)$ of NRCB1 follow two quadratic slopes with two plateaus as indicated in the graph. The slope at high strain amplitude falls into the region similar as the unfilled NR network. This implies that the nonlinearity of the composite could be a superposition of the filler network and the polymer network. Therefore, equation (6) can be modified into a function with two critical strain amplitudes to describe the $I_{3 / 1}\left(\gamma_{0}\right)$ of mixed networks:

$$
I_{3 / 1}\left(\gamma_{0}\right)=\frac{L_{\text {noise }}}{\gamma_{0}}+n_{F} * \frac{\left(\frac{\gamma_{0}}{\gamma_{c F}}\right)^{2}}{1+\left(\frac{\gamma_{0}}{\gamma_{C F}}\right)^{2}}+n_{P} * \frac{\left(\frac{\gamma_{0}}{\gamma_{C P}}\right)^{2}}{1+\left(\frac{\gamma_{0}}{\gamma_{c P}}\right)^{2}},
$$

where the $n_{F}$ and $n_{P}$ are the utmost nonlinearity, $\gamma_{c F}$ and $\gamma_{c P}$ are the critical strain amplitudes, with the indices " $F$ " and "P" representing the filler network and the polymer network, respectively.

Equations (6) and (7) fit quite well on both the experimental data of $I_{3 / 1}\left(\gamma_{0}\right)$ from the unfilled and the filled samples, as shown by the black lines in Fig. 3. For the $I_{3 / 1}\left(\gamma_{0}\right)$ of NRCB1, the filler network term (blue dot line with $\gamma_{c F}$ and $n_{F}$ ) and the polymer network term (green dash-dot line with $\gamma_{c P}$ and $n_{P}$ ) from the fitting results were presented in Fig. 3 as well. The fitting parameters of the filled and the unfilled NR were compared in Table 2. It has been found that within the same polymer matrix, both samples share the similar critical strain amplitude of the polymer network $\left(\gamma_{c P} \approx 70 \%\right)$ and similar level of the utmost nonlinearity ( $\left.n_{P}=0.049-0.065\right)$. The critical strain amplitude of the filler network for NRCB1 was around $\gamma_{C F} \approx 2.5 \%$.

Based on the study above, the rheological nonlinearity characteristics of crosslinked NR networks with and without filler can be separately analyzed. In following study on rubber aging, the nonlinearity at the critical strain amplitudes, $I_{3 / 1}\left(\gamma_{o}=2.5 \%\right)$ and $I_{3 / 1}\left(\gamma_{o}=70 \%\right)$, are 


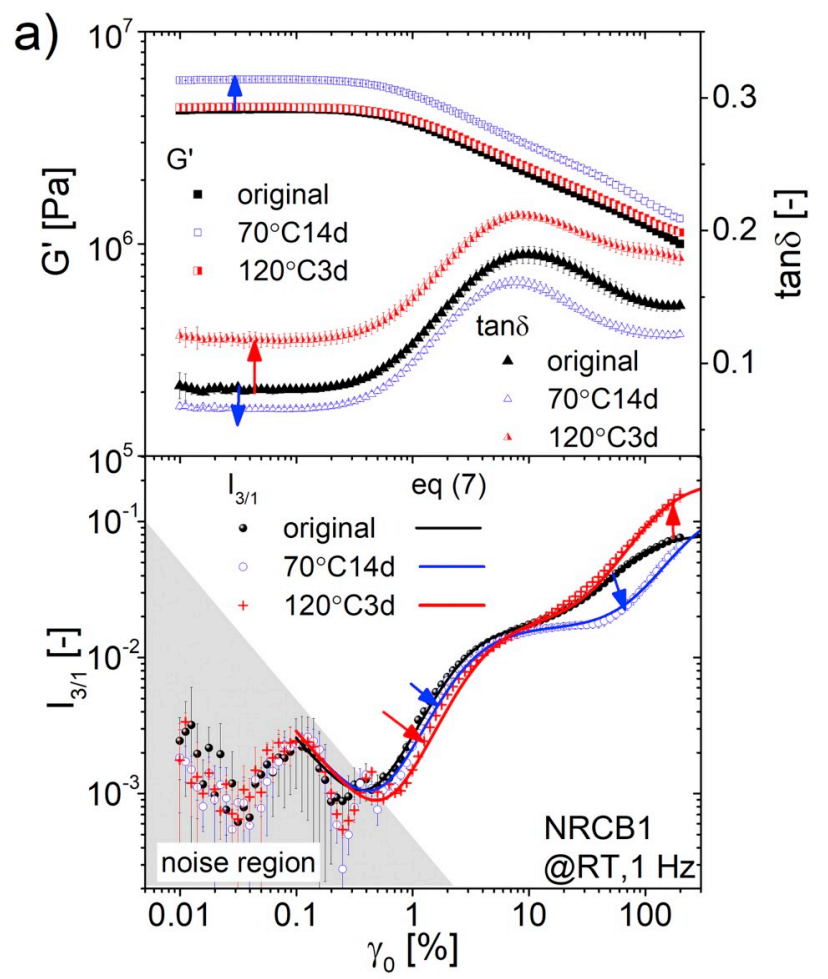

b)

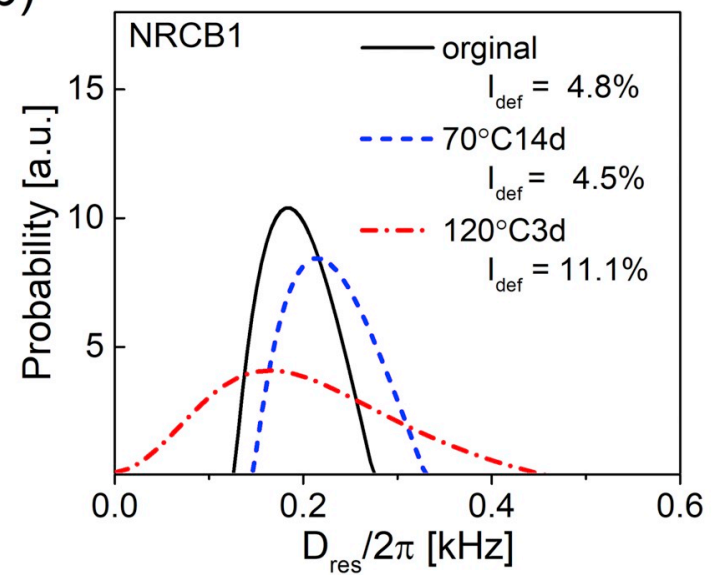

Fig. 8. The comparison of a) rheological properties and b) the polymer network structures of the crosslinked NR filled with carbon black (NRCB1) before and after aging in hot air at two typical conditions, $70{ }^{\circ} \mathrm{C}$ for 14 days and $120^{\circ} \mathrm{C}$ for 3 days.

selected for comparison, representing the rheological nonlinearity from the filler and polymer networks, respectively.

\subsection{DQ-NMR of NR vulcanizate}

In a polymer network, topological constraints (entanglements) and the permanent crosslinks hindered the movement of chain segments. Thus, the segmental fluctuations are anisotropic at long times and local residual order persists. This leads to a long-time plateau in the orientation autocorrelation function of the polymer chains. The plateau value is governed by the segmental dynamic order parameter of the polymer backbone [29], $S_{b}$, described $[43,44]$ by the average of the second order Legendre polynomial,

$S_{b}=\frac{1}{2}\left[3 \cos ^{2}(\theta)-1\right]=\frac{3}{5}\left(\frac{l R}{R_{0}^{2}}\right)^{2}=\frac{3}{5 N} \propto v$, with $\theta$ denoting the segmental orientation direction with respect to the end-to-end vector, $\vec{R}$. Here, $l$ is the length of the statistical chain segment (the freely jointed Kuhn segment) and $N$ is the number of such segments between two constraints. For bulk samples, $\left(R / R_{0}^{2}\right)^{2}=1 /\left(N l^{2}\right)$, leading to that $S_{b}$ is proportional to the effective network density, $\nu$.

The proton dipolar coupling is determined by the chain orientation and can be measured by DQ-NMR in term of the residual dipolar coupling constant, $D_{\text {res }}$. This is given by

$D_{\text {res }}=D_{e f f} S_{b} \propto v$,

with the $D_{\text {eff }}$ as the effective static limit of the dipolar coupling constant, which is fixed for a specified polymer. Overall, $D_{\text {res }}$ from ${ }^{1} \mathrm{H}$ DQ-NMR can represent the effective crosslink density of the polymer network.

Fig. 4 demonstrates the data treatment procedure $[30,38]$ to get the crosslink density and content of network defects, using the example of crosslinked NR. During the DQ-NMR measurement, two sets of magnetization intensity have been detected, the reference decay intensity, $I_{r e f}$, and the double quantum build-up intensity, $I_{D Q}$, as a function of the double quantum evolution time, $\tau_{D Q}$. The sum of the intensity $I_{D Q}+I_{r e f}$ is the full magnetization of the sample, containing the signal from dipolar-coupled network segments as well as the uncoupled parts. The uncoupled segments are the non-elastic network defects, including the dangling chains, crosslink loops, sol components, etc., which are isotropically mobile thus no observable dipolar coupling signal. In other words, the contribution of the network defects only exists in the reference signal $I_{\text {ref }}$, rather than in the $I_{D Q}$. In long time limit of $\tau_{D Q}, I_{D Q}$ should be equal to $I_{\text {ref }}$ without the contribution of the defects. Therefore, the long-term tail in the $I_{r e f}\left(\tau_{D Q}\right)-I_{D Q}\left(\tau_{D Q}\right)$ curve in Fig. 4a can be recognized as the contribution of the defects, $I_{\text {def }}$, obtained from the fitting with a single exponential function.

The total DQ magnetization is therefore $I_{D Q}+I_{r e f}-I_{\text {def. }}$. The normalized DQ intensity $I_{n D Q}=I_{D Q} /\left(I_{D Q}+I_{r e f}-I_{d e f}\right)$ in Fig. $4 \mathrm{~b}$, is independent of temperature at $\mathrm{T}>T_{g}+100 \mathrm{~K}$. At long $\tau_{D Q}, I_{n D Q}$ should reach a plateau value of 0.5 since it only contains half of the excited quantum orders. This $I_{n D Q}\left(\tau_{D Q}\right)$ can be submitted to a Tikhonov regularization program with a kernel fitting function described [38] as following,

$\mathrm{I}_{\mathrm{nDQ}}\left(\tau_{\mathrm{DQ}}, \mathrm{D}_{\text {res }}\right)=0.5\left\{1-\exp \left[-\left(0.378 \mathrm{D}_{\text {res }} \tau_{\mathrm{DQ}}\right)^{1.5}\right] \cos \left(0.583 \mathrm{D}_{\text {res }} \tau_{\mathrm{DQ}}\right)\right\}(10)$

The resulting distribution of $D_{\text {res }}$ (in unit of $\mathrm{kHz}$ for $D_{\text {res }} / 2 \pi$ if $\tau_{D Q}$ is in unit of $\mathrm{ms}$ ) from the regularization in Fig. $4 \mathrm{c}$ can represent the distribution of the crosslink density. High $D_{\text {res }}$ coupling rates indicate dense network and vice versa.

In summary, the network structure of NR vulcanizates can be quantitatively described by the crosslink density distribution in term of the residual dipolar coupling constant, $D_{\text {res }}$, and the content of network defects, $I_{\text {def }}$, through DQ-NMR measurement.

\subsection{Hot air aging}

\subsubsection{Aging of unfilled $N R$}

The thermal oxidation effect on the crosslinked NR without fillers was studied first. Fig. 5 presents rheological properties of NR1 before and after aerobic aging at two typical conditions, $70{ }^{\circ} \mathrm{C}$ for 14 days and $120^{\circ} \mathrm{C}$ for 3 days, respectively. After being exposed to hot air at $70^{\circ} \mathrm{C}$ for 14 days, NR1 changed into a more elastic material with lower $\tan \delta$. After $120^{\circ} \mathrm{C}$ for 3 days in hot air, $\tan \delta$ of the network strongly increased. The rheological nonlinearity, $I_{3 / 1}$ of the $70^{\circ} \mathrm{C}$ for 14 days aged sample declined (after the noise region).

As discussed in chapter 3.1, the storage modulus and loss factor at linear regime, $G^{\prime}{ }_{0}$ and $\tan \delta_{0}$, were chosen as the linear rheological parameters for the comparison between aged and unaged samples. With respect to the nonlinear rheological parameters, $I_{3 / 1}$ at $\gamma_{0}=70 \%$ were selected to present the characteristic nonlinearity of the polymer network (while $I_{3 / 1}$ at $\gamma_{0}=2.5 \%$ for the filler networks of the filled 

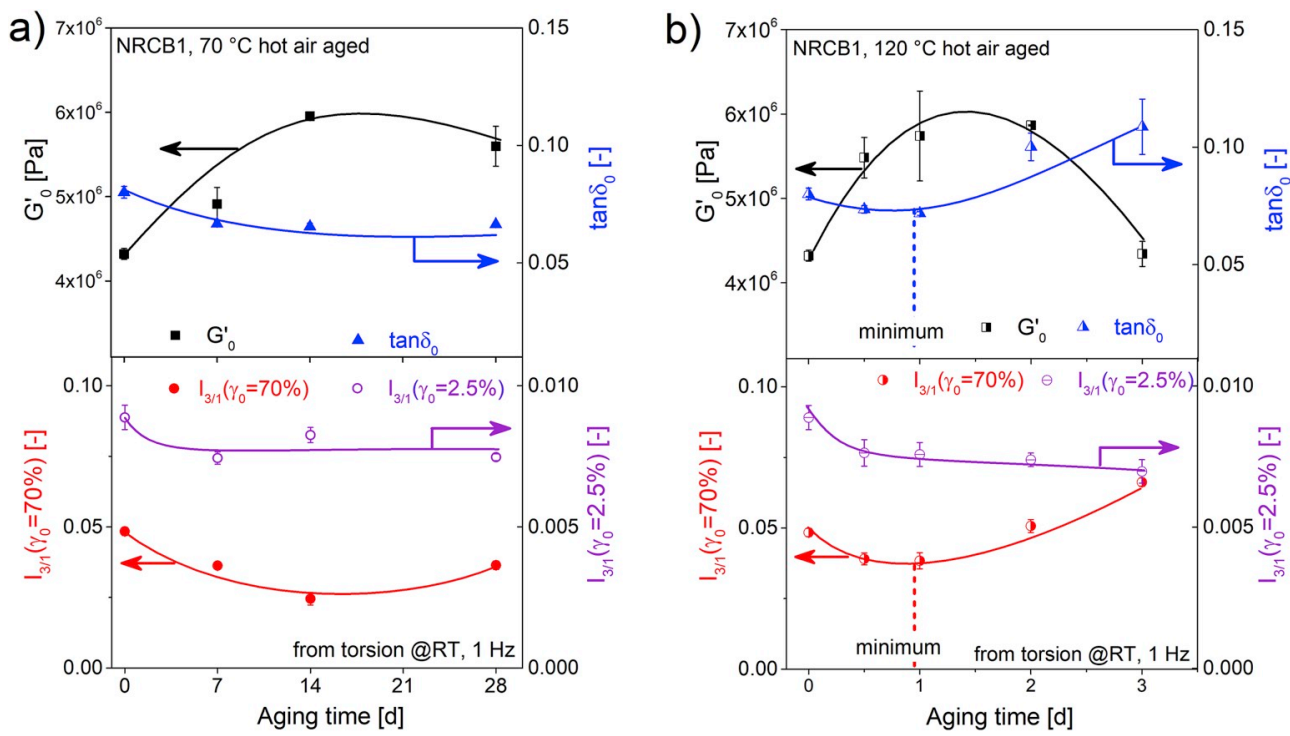

Fig. 9. The evolution of the rheological parameters, $G^{\prime}{ }_{0}$, $\tan \delta_{0}$ and $I_{3 / 1}\left(\gamma_{0}=70 \%\right)$, of NRCB1 at progressive aging in air at a) $70{ }^{\circ} \mathrm{C}$ and b) $120{ }^{\circ} \mathrm{C}$, respectively. The rheological parameters were obtained from oscillatory torsion at room temperature and $1 \mathrm{~Hz}$. The lines in the figure were drawn as the guide to the eye.

samples in following section). The evolution of those parameters as well as the crosslink structures in time during hot air aging at different temperatures are depicted in Fig. 6.

During the hot air aging test at $70{ }^{\circ} \mathrm{C}$ in 28 days, the $G^{\prime}{ }_{0}$ of NR vulcanizate mainly increased along the aging time and $\tan \delta_{0}$ decreased as shown in Fig. 6a. This was complemented by a decrease of the rheological nonlinearity as indicated by $I_{3 / 1}\left(\gamma_{0}=70 \%\right)$. The development of those rheological properties slowed down after 14 days. Accordingly, the change of the network structures were revealed by the DQ-NMR results in Fig. 6c. During aerobic aging at $70^{\circ} \mathrm{C}$, higher dipolar-coupled network was produced without the rise of $D_{\text {res }}$ at low rates, indicating that only denser networks were formed. This is consistent with other studies $[8,9]$ of the thermally aged NR vulcanizate that the polysulfidic crosslinks in the original sample can transform into short linkages as mono- or disulfide bonds, resulting in higher network density. Furthermore, the amount of the network defects, $I_{d e f}$ maintained the original level or even slightly reduced. This suggests that the chain scission reactions rarely occurred during the $70^{\circ} \mathrm{C}$ aerobic aging.

The crosslink density $\nu$ of a network directly affects its elasticity. The storage modulus at equilibrium $G_{e}$ is proportional to the crosslink density as $G_{e}=\nu R T$ [13], where $R$ is the universal gas constant. Therefore, the increase of crosslink density of NR after aging resulted in higher elasticity $G^{\prime}{ }_{0}$. The more elastic network with fewer defects resulted in a decrease of $\tan \delta_{0}$. In addition, the decrease of $I_{3 / 1}\left(\gamma_{0}=70 \%\right)$ after aging suggested that higher density network with fewer defects would be rheologically less nonlinear. One possible explanation could be that denser network would more tightly constrain the movement of the chain segments, which are rheologically nonlinear, thus weaken their nonlinear response to the external deformation.

With respect to progressive hot air aging of NR1 at $120^{\circ} \mathrm{C}$, there likely exist a transition point at around 0.5-1 day for the change of the rheological properties as indicated by the dash lines in Fig. $6 \mathrm{~b}$. The storage moduli increased at the earlier stage and then declined. It was accompanied by a decrease of $\tan \delta_{O}$ firstly and then a drastic rise after 0.5 day with the same manner of the nonlinear parameter, $I_{3 / 1}\left(\gamma_{0}=\right.$ $70 \%$ ). The evolution of the crosslink density and the content of defects at this aging condition was shown in Fig. 6d. Corresponding to the reversing trend of the rheological properties as a function of aging time, the distribution curve of $D_{\text {res }}$ also clearly turned over at around 0.5-1 day. Before 1 day, there were only highly dipolar-coupled network generated, which was similar to the $70^{\circ} \mathrm{C}$ aging process within 14 days in Fig. 6c. This led to the rise of the elasticity and the decrease of nonlinearity at the beginning period of aging. After 1 day, $D_{\text {res }}$ mainly shifted towards lower coupling rates with a broader distribution. Additionally, the content of network defects had been more than doubled from $I_{r e f}=5.3 \%$ of the original to $I_{r e f}=11.5 \%$ of the 3 days aged. This loose network with high content of defects should be responsible for the deterioration of the linear rheological properties and also the increase of nonlinearity after 0.5 day.

Based on the aging results in Fig. 6, the evolution scheme of sulfurcured NR network structure in hot air at the two elevated temperatures can be described in Fig. 7. Before 14 days at $70{ }^{\circ} \mathrm{C}$, or 0.5 days at $120^{\circ} \mathrm{C}$, the polysulfidic crosslinks in the starting material transform into short linkages as mono- or disulfide bonds [8,9], forming a network with denser crosslinks. Meanwhile, there are rarely network defects generated yet. With longer aging time, the network scission reactions start to take place on the polymer chains as well as at the sulfur linkages [7,27], resulting in network defects with alcohol and carbonyl groups $[27,45,46]$ due to thermal oxidation. At the same time, a small portion of the sulfidic crosslinks could also transform into cyclic sulfides [7], creating highly constrained networks $\left(D_{\text {res }} / 2 \pi\right.$ at ca. $\left.0.4 \mathrm{kHz}\right)$. However, the scission reactions still dominate and overall a network with broader mesh size distribution is resulted in. These structural changes of the network at different aging periods affect the rheological properties accordingly, see above.

\subsubsection{Aging of filled NR}

Most crosslinked rubbers for engineering products are used not solely but rather filled with organic fillers to improve the mechanical properties. By mixing highly active fillers into the rubber matrix, the products can achieve higher mechanical strength from the filler networks, an effect called reinforcement. Both the linear and nonlinear rheological parameters of NR networks with and without CB before aging have been compared in section 3.1 to emphasize the differences between the filled and unfilled system. It should be pointed out that there are two critical strain amplitudes for the development of $I_{3 / 1}\left(\gamma_{0}\right)$ of the filled rubber. The $I_{3 / 1}\left(\gamma_{0}\right)$ of NRCB1 grows quadratically at both $\gamma_{o}=2.5 \%$ and $\gamma_{o}=70 \%$, which has been considered as the origination from filler network and polymer network, respectively. Following studies present the aging phenomena of CB filled NR vulcanizates.

Regarding the hot air aging of NRCB1, Fig. 8 displayed the changes of rheological properties as well as the polymer network structures after aging at two typical conditions, $70^{\circ} \mathrm{C}$ for 14 days and $120^{\circ} \mathrm{C}$ for 3 days.

After $70^{\circ} \mathrm{C}$ for 14 days in air, the network segments mainly shifted 

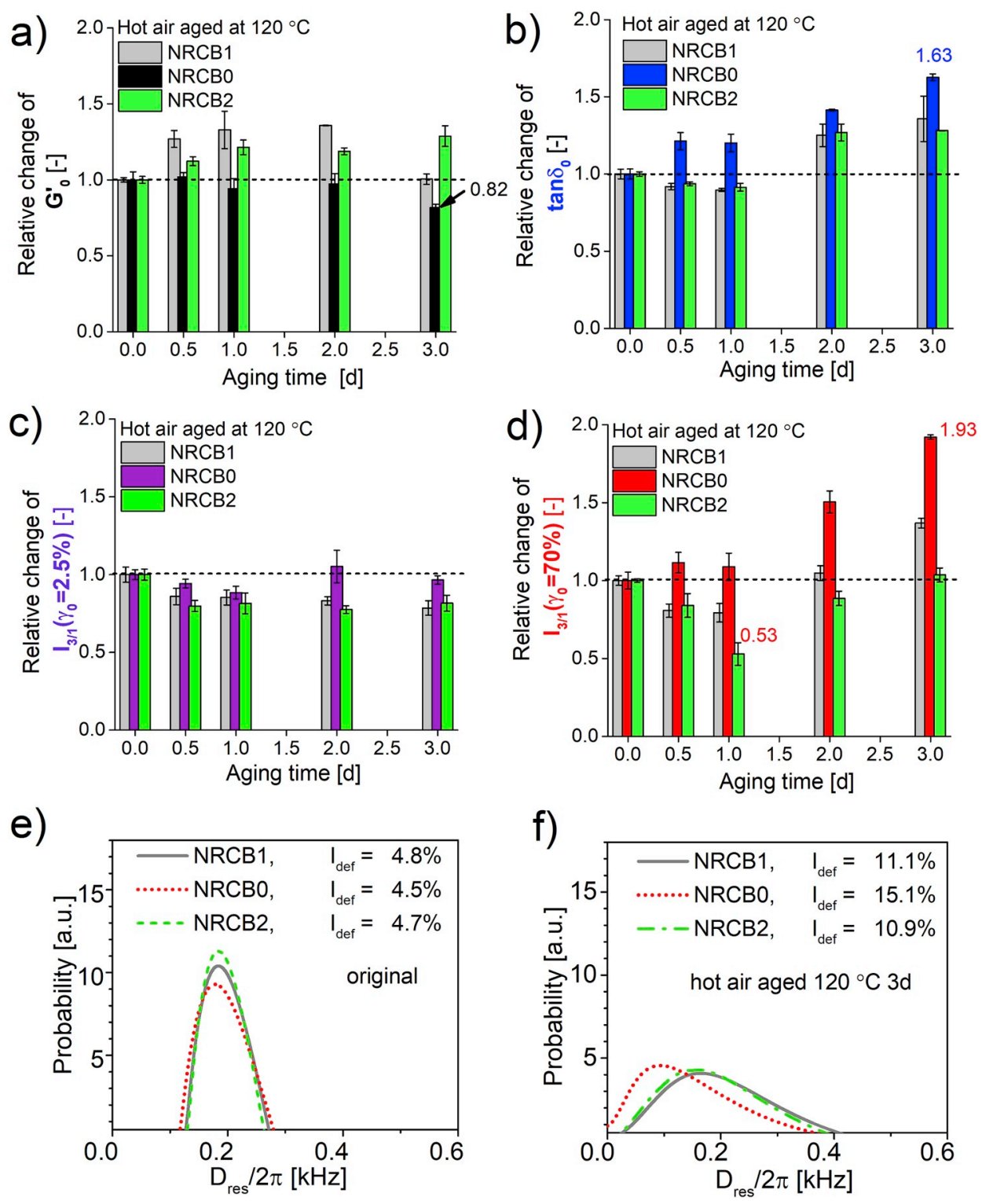

Fig. 10. The relative change of the rheological parameters a) $G^{\prime}{ }_{0}$, b) $\tan \delta_{0}$, c) $I_{3 / 1}\left(\gamma_{o}=2.5 \%\right)$, and d) $I_{3 / 1}\left(\gamma_{o}=70 \%\right)$ of the samples with different amount of antioxidant (NRCB1, NRCB0, NRCB2) after hot air aging at $120^{\circ} \mathrm{C}$. The rheological parameters were obtained from oscillatory torsion at room temperature and $1 \mathrm{~Hz}$. The relative change was calculated by normalizing the sample values to their unaged status, indicated by dashed lines. The distribution of $D_{\text {res }}$ and the amount of network defects $I_{\text {ref }}$ of e) the original samples and f) the hot air aged at $120^{\circ} \mathrm{C}$ at 3 days were selected for comparison.

to higher dipolar-coupled ones with no extra generation of network defects. This led to the increase of elasticity and the lowering of $\tan \delta$. The nonlinear parameter $I_{3 / 1}$ decreased at large strain amplitude as indicated by the blue arrow in the graph. After the hot air aging at $120^{\circ} \mathrm{C}$ for 3 days, the distribution of $D_{\text {res }}$ distinctively broadened with the curve shifted to the lower coupling rates (lose network). Furthermore, the content of network defects was more than doubled after aging. This resulted in a rise in $\tan \delta$ and an increase in the nonlinearity. The change of polymer network structure as well as the rheological properties after hot air aging at those two conditions were similar to the unfilled sample as discussed in last section.

The progressive hot air aging of NRCB1 at the two elevated temperatures were shown in Fig. 9. Compared to the $70{ }^{\circ} \mathrm{C}$ aerobic aging process of the unfilled vulcanizate, NR1 in Fig. $6 \mathrm{a}$, there were no obvious differences for the aging course of NRCB1. However, regarding to the aging process at $120^{\circ} \mathrm{C}$ (Fig. $6 \mathrm{~b}$ versus Fig. 9b), the evolution of the rheological parameters, $G^{\prime}{ }_{0}, \tan \delta_{O}$ and $I_{3 / 1}\left(\gamma_{O}=70 \%\right)$, were delayed in NRCB1. The transition points (the minimum as indicated by the dashed lines) of $\tan \delta_{O}$ and $I_{3 / 1}\left(\gamma_{O}=70 \%\right)$ for NRCB1 was half days later than that for NR1. This may due to the filled CB, acting as a free radical acceptor, which could absorb the resulting free radical at high temperature thus slow down the oxidation process of the polymer chains. It is also suggested [47] that the thermal stability of the rubber composites could be enhanced in the case of good filler-polymer interaction and improved thermal conductivity.

In addition, the nonlinearity at medium strain amplitude $I_{3 /}$ ${ }_{1}\left(\gamma_{0}=2.5 \%\right)$, representing the contribution from filler network, decreased for all progressively aged samples to a similar level at both temperatures, as shown in Fig. 9. The decrease of the filler network nonlinearity after hot air treatment was expected, since at elevated temperatures the reversible part of bound rubber could desorb from the surface of the filler [48-50]. The disconnection of filler network from polymer network could result in the decrease of nonlinearity of the filler network.

To protect the rubber materials against thermal oxidation, antiaging agent is commonly added into the compounds during mixing. 

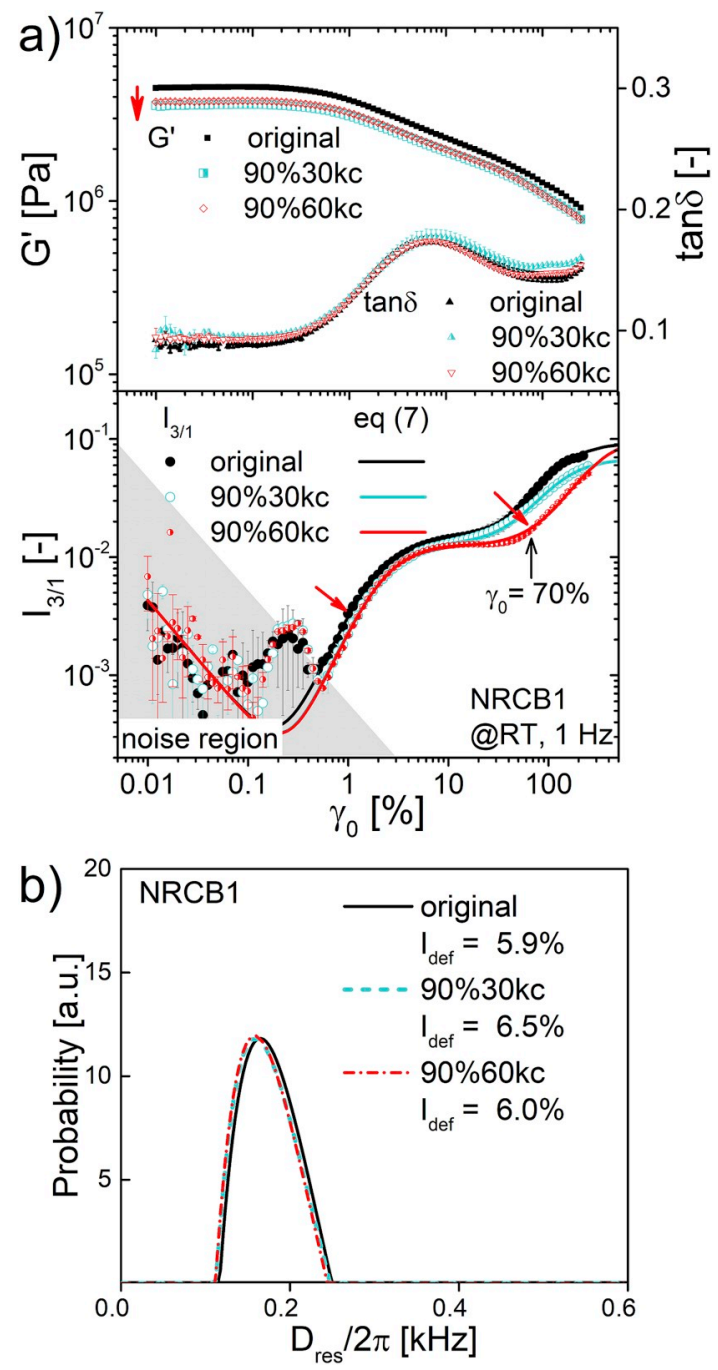

Fig. 11. The comparison of a) rheological properties and b) the polymer network structures as analyzed via ${ }^{1} \mathrm{H}$ DQ-NMR of NRCB1 before and after fatigue by stretching to $\varepsilon=90 \%$ for 30 or 60 kilocycles.

NRCB1 were mixed with normal dosage (1.8 phr here) of anti-oxidant to simulate the tire tread compounds. Two extra vulcanizates without and with double dosage of anti-oxidant were prepared, named as NRCB0 and NRCB2, respectively. The progressive aerobic aging at $120^{\circ} \mathrm{C}$ of those compounds were compared in Fig. 10a-d with respect to their rheological properties. All of the rheological parameters were normalized by the values of their unaged state (the relative change or the retention after aging) for comparison in order to eliminate the differences between the starting samples. The distribution of $D_{\text {res }}$ and network defects content $I_{\text {def }}$ of those three samples before and after hot air treatment at $120^{\circ} \mathrm{C}$ for 3 days were selected for comparison in Fig. 10e and $\mathrm{f}$.

Without adding the anti-oxidant, there was no increase of elasticity, decrease of $\tan \delta_{0}$ and $I_{3 / 1}\left(\gamma_{o}=70 \%\right)$ for NRCB0 at the early aging period ( $<2$ days) at $120^{\circ} \mathrm{C}$ as NRCB1 and NRCB2. At long aging time, $\tan \delta_{O}$ and $I_{3 / 1}\left(\gamma_{0}=70 \%\right)$ of NRCB0 increased more stongly than that of NRCB1. This indicated that the anti-oxidant had already taken effect in the early aging period at $120^{\circ} \mathrm{C}$. For the sample with double amount of anti-oxidant, NRCB2, the relative change of elasticity got depressed, comparing to that of NRCB1. In addition, its increase of $I_{3 / 1}\left(\gamma_{0}=70 \%\right)$ after $120^{\circ} \mathrm{C}$ for 2 days was not as severe as NRCB1. This suggested that double the anti-oxidant dosage would be helpful for hot air conditions at $120^{\circ} \mathrm{C}$ after 2 days. After 3 days aerobic aging at $120^{\circ} \mathrm{C}$, the polymer network of NRCB0 changed more dramatically than other two samples with higher portion of loose network and 5\% more defects. Nevertheless, the change of polymer network for NRCB2 after hot air aging at $120^{\circ} \mathrm{C}$ for 3 days was similar as that for NRCB1.

To compare the relative sensitivity of those rheological parameters to hot air aging at $120^{\circ} \mathrm{C}$, the distinctive relative changes are labelled by the values in Fig. 10. The most relative change of linear rheological parameters were the $18 \%$ decrease of the $G^{\prime}{ }_{0}$ and the $63 \%$ increase of $\tan \delta_{0}$ from the aged NRCB0 at 3 days. Comparatively, $I_{3 / 1}\left(\gamma_{0}=70 \%\right)$ exhibited more pronounced relative change, with an amplitude of $47 \%$ decrease from the 1 day aged NRCB2 and 93\% increase from the 3 days aged NRCBO. All in all, both the lowest and highest relative change of rheological properties after hot air aging were indicated by this nonlinear parameter, $I_{3 / 1}\left(\gamma_{0}=70 \%\right)$, within the tested aging conditions and samples.

In this section, the evolution of rheological nonlinearity as well as the crosslink structure of NR vulcanizate under progressive hot air aging has been studied. Before aging at $70^{\circ} \mathrm{C}$ in 14 days, or at $120^{\circ} \mathrm{C}$ in 0.5 days, the network density becomes higher thus results in the decrease of nonlinearity. Afterwards, the mesh size of the network turns into broader distribution with more defects, which are responsible for the increase of the nonlinearity. Carbon black as a filler can delay the thermal-oxidation aging effect on NR by 0.5 day at $120^{\circ} \mathrm{C}$. Within the tested samples and conditions, the parameter from FT-Rheology, $I_{3 /}$ ${ }_{1}\left(\gamma_{0}=70 \%\right)$, exhibits higher relative sensitivity to the hot air aging effect, compared to the linear rheological parameters.

\subsection{Mechanical aging}

Apart from the static aging conditions as above, crosslinked NR products also widely endure mechanical aging under large deformation or dynamic loading of stress, e.g. the running tires and the conveying belts. This will result in the fatigue of the materials. Therefore, mechanical aging behavior of NR was also studied with the sample of NRCB1 at different fatigue cycles but before failure.

The dumbbell specimens of NRCB1 were stretched to $\varepsilon=90 \%$ for 30 or 60 kilocycles, labelled as NRCB1-90\%30kc and NRCB1-90\%60kc, respectively. During the fatigue test, there were still neither visually observable cracks, nor a drastic drop of the modulus $(<50 \%$ drop of the Yong's modulus at the first cycle) which could indicate a failure. The working area of the fatigued dumbbells were selected for the specimen preparation of rheological, DQ-NMR and microscopic measurements.

The rheological properties and polymer network structure of the mechanically aged samples are compared in Fig. 11. The $G^{\prime}$ only slightly dropped after fatigue and the tan $\delta$ barely changed within the graph. Moreover, there was no detectable effect from the number of fatigue cycles on the linear rheological parameters. On the contrary, the nonlinear parameter $I_{3 / 1}$ decreased most pronouncedly at large strain amplitude (around $\gamma_{o}=70 \%$ ) as a function of the fatigue cycle numbers. The decrease of $I_{3 / 1}$ as function of cycle numbers were also observed from polystyrene materials [51] during a continuous fatigue test at fixed strain amplitude and oscillatory frequency. The $I_{3 / 1}$ (cycle numbers) decreased [51] to a minimum value before failure with the propagation of the crack in the specimen.

The nonlinearity at large strain are considered to be the origination from the polymer network as discussed in section 3.1. Whereas, the DQNMR results in Fig. 11b indicated neither obvious change of the crosslink density and nor of the amount of network defects. With the aid of light microscope, the fatigued samples and the original can be distinguished from the micrographs as compared in Fig. 12a-c. Cracks with a length of around $50 \mu \mathrm{m}$ had been found in the sample fatigued at 60 kilocycles, which was not the case for the unaged. Artifacts can also be excluded in the case of the micro-cracks because they appear independently of the cutting striations caused by the irregularities of the scalpel blade. The micro-cracks in the specimen may changed the mechanical anisotropy of the material [52]. Under strain, samples with those micrometer size cracks could arrange the inter-deformation more 

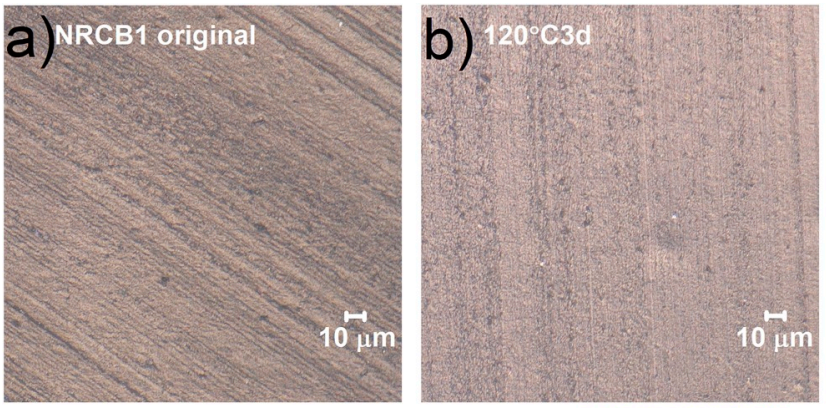

d)

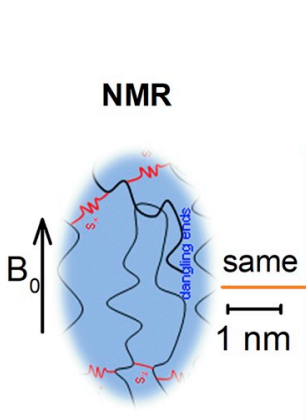

adjacent defects
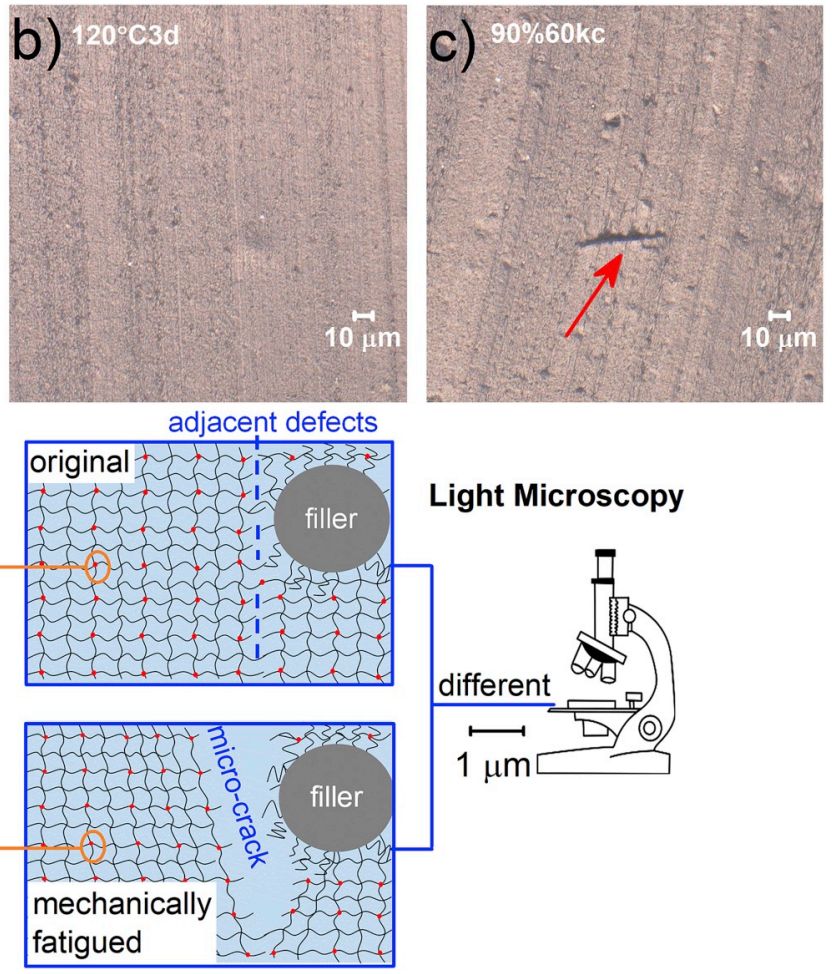

\section{Light Microscopy}

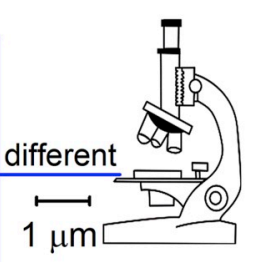

Fig. 12. The cross-section of NRCB vulcanizate a) at its original state, b) aged statically in hot air at $120^{\circ} \mathrm{C}$ for 3 days and c) fatigued at $\varepsilon=90 \%$ for 60 kilo cycles, under light reflection microscopy at a magnification of $1000 \times$. Lines across the images are cutting striations, a preparation artifact. d) Schematic illustration of the network structures in the vulcanizate before and after mechanical fatigue. The nanometer scale crosslinks in polymer matrix on average would not change thus not be detectable by DQ-NMR. Whereas the micrometer size cracks generated from the adjacent network defects during fatigue can be observed by optical microscopy.
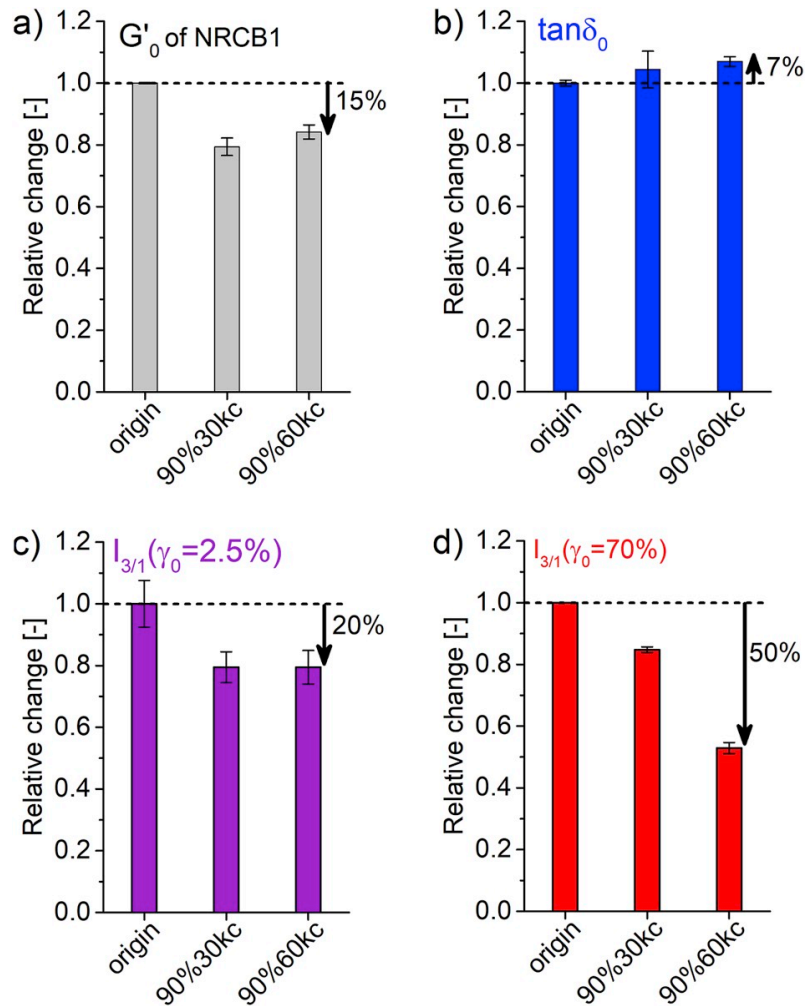

Fig. 13. The relative change of the rheological parameters a) $G_{0}^{\prime}$, b) $\tan \delta_{0}$, c) $I_{3 /}$ ${ }_{1}\left(\gamma_{o}=2.5 \%\right)$ and $\left.\mathrm{d}\right) I_{3 / 1}\left(\gamma_{o}=70 \%\right)$ of the fatigued samples (NRCB1 at $\varepsilon=90 \%$ for 30 or 60 kilo cycles). The parameters were obtained from oscillatory torsion at room temperature and $1 \mathrm{~Hz}$. The relative change value were calculated by normalizing to the original samples' values (as the dashed lines to guide the eye).

easily thus the overall stress response is less nonlinear.

Although there were structural changes of the polymer network in hot air aged NRCB1, as depicted with DQ-NMR in section 3.3, there were also no micro-cracks revealed by the light microscopy of those statically aged samples as shown in Fig. 12b. The spatial detection limit of a light microscope is about $1 \mu \mathrm{m}$, while the polymer network change measured by DQ-NMR technique occurs at a length scale of around $1 \mathrm{~nm}$ or below. Interestingly, based on the DQ-NMR results in Fig. 11b, there were no detectable differences between the original sample and the mechanically aged sample with micro-cracks. This suggests that, after mechanical aging, the localized (nanometer scale) polymer network structure on average does not change, since the DQ-NMR results are the mean values of the polymer chain dynamics in nanometer scale of the overall sample.

It could be reasonable to sketch the network change before and after mechanical aging as Fig. 12d. Only the localized (nanometer scale, as the brown cycles in the graph) polymer network structure can be represented by DQ-NMR. However, the structural change above $1 \mu \mathrm{m}$, such as a micro-crack as indicated in the graph, can be observed with the light microscope. In the original sample, there could be some crosslinking flaws or other defects, which are by chance close to each other or near a filler aggregate. Compared to the main polymer matrix, the polymer chains in this area are much less bonded by the sulfidic crosslinks and mainly physically entangled with neighbour dangling ends or adsorbed on the active filler surface. Once an external force is applied on the sample, stresses would concentrate between the soft area with defects (softer than the average of polymer matrix) and the rigid filler. During the energy dissipation for the cyclically loaded force, the trace amount of crosslinks as well as the transient entanglements between the adjacent defects could be gradually broken up or disentangled along one direction. Finally, a continuous crack in micrometer size could appear or be enlarged. However, the chemical crosslink density in the polymer matrix would not change on average since the broken chemical bonds along the cracks are trivial amount. Nevertheless, both the nanometer structural change in NR vulcanizates, e.g. hot air aged samples in section 3.3, and the micrometer structural change of the NR composites, e.g. the mechanically aged in this section, 
can be monitored by FT-rheology.

Although the micro-cracks did not cause strong deterioration of the linear rheological properties during mechanical aging, they did result in distinctive change of the nonlinearity at large strain, as observed in Fig. 11a. The relative change of the rheological parameters after mechanical aging was compared in Fig. 13. It is found that $I_{3 / 1}\left(\gamma_{o}=70 \%\right)$ was far more sensitive to the mechanical aging of rubber (with a relative change of 50\%) than linear rheological parameters as ${G^{\prime}}_{0}$ or $\tan \delta_{0}$ ( $15 \%$ and $7 \%$ relative change for ${G^{\prime}}_{o}$ and $\tan \delta_{0}$, respectively).

The nonlinearity of the filler involved network, $I_{3 / 1}\left(\gamma_{0}=2.5 \%\right)$, decreased for NRCB1 after mechanical aging. This could result from the desorption of the polymer chains from the filler surface during mechanical aging. In addition, the decrease of $I_{3 / 1}\left(\gamma_{0}=2.5 \%\right)$ in amplitude for NRCB1 fatigued at 30 and 60 kilocycles was similar. This provides a hint that there is certain amount of reversible polymer chains which could be desorbed from the filler for the same original vulcanizate.

During hot air aging of NR in section 3, the structural changes of the materials mainly resulted from the chemical reactions of the vulcanized polymer networks, including the change of sulfidic crosslinks and the chain scission of the polymer backbones via thermo-oxidation. This will lead to a decrease of the rheological nonlinearity of the polymer network, e.g. with higher crosslink density, or an increase of the rheological nonlinearity, e.g. due to high content of network defects. However, during mechanical aging, the physical disentanglements dominate along the network flaws instead of chemical modifications of the polymer matrix. This will result in a progressive decrease of $I_{3 /}$ ${ }_{1}\left(\gamma_{0}=70 \%\right)$ as a function of the fatigue cycle numbers.

Limitations for analytical tools to monitor the mechanical aging of crosslinked rubber materials are: 1 ) DQ-NMR technique can not reveal macroscopic changes of the samples as the average local $(<10 \mathrm{~nm})$ crosslinked structures stays the same; 2) the generated micro-cracks, which are not visually observable from the material surface, can only be qualitatively probed under the microscope by cutting the materials into the internal section; 3) the linear rheological method is relatively insensitive. Therefore, $I_{3 / 1}$ at large strain amplitude is considered to be a more effective and quantitative parameter for the determination of mechanical aging.

\section{Conclusions}

In this work, the aging process of crosslinked NR in hot air or under mechanical fatigue has been examined. With FT-Rheology, the rheological nonlinearity has been directly quantified in term of $I_{3 / 1}$, and with DQ-NMR, the distribution of the network density as well as the amount of defects have been revealed.

During hot air aging, the network density continuously increases without generation of defects at $70^{\circ} \mathrm{C}$ in 28 days. This results in an increase of elasticity and decrease of rheological nonlinearity. At $120^{\circ} \mathrm{C}$, the distribution of network density becomes broad with more defects after 1 day. This is responsible to the deterioration of the linear rheological properties and the increase of the rheological nonlinearity. By filling with $50 \mathrm{phr} \mathrm{CB} \mathrm{N} 339$, the aging course of $\mathrm{NR}$ at $120^{\circ} \mathrm{C}$ is delayed by 0.5 days.

During mechanical aging, the local network structure of the matrix does not change according to DQ-NMR. However, there are already micro-cracks inside of the fatigued samples, which may originate from adjacent network flaws in the starting material. This leads to a slight decrease of elasticity and a continuous decline of $I_{3 / 1}\left(\gamma_{o}=70 \%\right)$ along the fatigue cycles.

Compared to the linear rheological parameters, $I_{3 / 1}\left(\gamma_{0}=70 \%\right)$ has exhibited higher relative sensitivity on the effect of hot air aging and, in particular, mechanical aging. With the limitations of DQ-NMR and microscope to detect the aging effect, $I_{3 / 1}$ can respond to the structural changes of the material at both nano- and micrometer scales. Therefore, FT-Rheology could be a valuable tool to study the aging of rubber. On one hand, it provides the more effective parameter $I_{3 / 1}$, and on the other, it brings more insight into the rheological nonlinearity of the material.

\section{Acknowledgements}

The financial support and sample preparation from Continental Reifen Deutschland GmbH are gratefully acknowledged. The authors are indebted to Dr. Frank Fleck, Dr. Anna-Lena Dreier and Dr. Christoph Vatterott from Continental Reifen Deutschland GmbH for helpful discussions. S.N. thanks Jonas Keller and Dr. Karl-Friedrich Ratzsch for generous support of the DQ-NMR measurement, Dr. Michael Pollard and Dr. Ciprian Iacob for the proofreading and valuable comments on the manuscript.

\section{References}

[1] C. Goodyear, Gum-elastic and its Varieties: with a Detailed Account of its Applications and Uses, and of the Discovery of Vulcanization, (1853) published for the author.

[2] H.L. Fisher, Vulcanization of rubber, Ind. Eng. Chem. 31 (1939) 1381-1389.

[3] Resources from International Rubber Study Group (IRSG), Rubber Statistical Bulletin, (2018).

[4] K. Nagdi, Rubber as an Engineering Material: Guideline for Users vol. 31, Hanse Verlag, 1993, pp. 38-98.

[5] J.I. Cunneen, Oxidative aging of natural rubber, Rubber Chem. Technol. 41 (1968) 182-208.

[6] G.J. Lake, P.B. Lindley, The mechanical fatigue limit for rubber, J. Appl. Polym. Sci. 9 (1965) 1233-1251.

[7] E.J. Blackman, E.B. McCall, Relationships between the structures of natural rubber vulcanizates and their thermal and oxidative aging, Rubber Chem. Technol. 43 (1970) 651-663.

[8] N.M. Mathew, S.K. De, Thermo-oxidative ageing and its effect on the network structure and fracture mode of natural rubber vulcanizates, Polymer 24 (1983) 1042-1054.

[9] R. Fan, Y. Zhang, C. Huang, Y. Zhang, Y. Fan, K. Sun, Effect of crosslink structures on dynamic mechanical properties of natural rubber vulcanizates under different aging conditions, J. Appl. Polym. Sci. 81 (2001) 710-718.

[10] G.Y. Li, J.L. Koenig, A review of rubber oxidation, Rubber Chem. Technol. 78 (2005) 355-390

[11] N.T. Thuong, Y. Yamamoto, P.T. Nghia, S. Kawahara, Analysis of damage in commercial natural rubber through NMR spectroscopy, Polym. Degrad. Stab. 123 (2016) 155-161.

[12] W. Grellmann, G. Heinrich, M. Kaliske, M. Klüppel, K. Schneider, T. Vilgis (Eds.), Fracture Mechanics and Statistical Mechanics of Reinforced Elastomeric Blends, Springer Science and Business Media, Berlin, 2013.

[13] J.D. Ferry, Viscoelastic Properties of Polymers, John Wiley and Sons, New York, 1980, p. 234.

[14] J.L. Leblanc, Filled Polymers - Science and Industrial Applications, CRC Press, Boca Raton, 2010, pp. 25-141.

[15] M. Wilhelm, D. Maring, H.W. Spiess, Fourier-transform rheology, Rheol. Acta 37 (1998) 399-405.

[16] M. Wilhelm, Fourier-transform rheology, Macromol. Mater. Eng. 287 (2002) 83-105.

[17] K. Hyun, M. Wilhelm, C.O. Klein, K.S. Cho, J.G. Nam, K.H. Ahn, S.J. Lee, R.H. Ewoldt, G.H. McKinley, A review of nonlinear oscillatory shear tests: analysis and application of large amplitude oscillatory shear (Laos), Prog. Polym. Sci. 36 (2011) 1697-1753.

[18] M.A. Cziep, M. Abbasi, M. Heck, L. Arens, M. Wilhelm, Effect of molecular weight, polydispersity, and monomer of linear homopolymer melts on the intrinsic mechanical nonlinearity ${ }^{3} \mathrm{Q}_{0}(\omega)$ in MAOS, Macromolecules 49 (2016) 3566-3579.

[19] D. Ahirwal, H. Palza, G. Schlatter, M. Wilhelm, New way to characterize the percolation threshold of polyethylene and carbon nanotube polymer composites using Fourier transform (FT) rheology, Korea Aust. Rheol. J. 26 (2014) 319-326.

[20] J.L. Leblanc, Effect of temperature on dynamic rheological properties of uncured rubber materials in both the linear and the nonlinear viscoelastic domains, J. Appl. Polym. Sci. 126 (2012) 408-422.

[21] L. Schwab, N. Hojdis, J. Lacayo-Pineda, M. Wilhelm, Fourier-transform rheology of unvulcanized, carbon black filled styrene butadiene rubber, Macromol. Mater. Eng. 301 (2016) 457-468.

[22] P.J. Flory, J. Rehner, Statistical mechanics of crosslinked polymer networks I. Rubberlike elasticity, J. Chem. Phys. 11 (1943) 512-520.

[23] C.G. Moore, B.R. Trego, Structural characterization of vulcanizates. Part IV. Use of triphenylphosphine and sodium di-n-butyl phosphite to determine the structures of 
sulfur linkages in natural rubber, cis-1, 4-polyisoprene, and ethylene-propylene rubber vulcanizate networks, J. Appl. Polym. Sci. 8 (1964) 1957-1983.

[24] B.A. Saville, A.A. Watson, Structural characterization of sulfur-vulcanized rubbe networks, Rubber Chem. Technol. 40 (1967) 100-148.

[25] W. Kuhn, E. Peterli, H. Majer, Freezing point depression of gels produced by high polymer network, J. Polym. Sci. 16 (1955) 539-548.

[26] D. Honiball, M.G. Huson, W.J. McGill, A nucleation theory for the anomalous freezing point depression of solvents in swollen rubber gels, J. Polym. Sci. B 26 (1988) 2413-2431.

[27] Y.R. Yahya, A.R. Azura, Z. Ahmad, Effect of curing systems on thermal degradation behaviour of natural rubber (SMR CV 60), J. Phys. Sci. 22 (2011) 1-14.

[28] J.Y. Buzaré, G. Silly, J. Emery, G. Boccaccio, E. Rouault, Aging effects on vulcanized natural rubber studied by high resolution solid state 13C-NMR, Eur. Polym. J. 37 (2001) 85-91.

[29] K. Saalwächter, Proton multiple-quantum NMR for the study of chain dynamics and structural constraints in polymeric soft materials, Prog. Nucl. Magn. Reson. Spectrosc. 51 (2007) 1-35.

[30] J.L. Valentín, P. Posadas, A. Fernández-Torres, M.A. Malmierca, L. González, W. Chassé, K. Saalwächter, Inhomogeneities and chain dynamics in diene rubbers vulcanized with different cure systems, Macromolecules 43 (2010) 4210-4222.

[31] V.M. Litvinov, P.P. De, Spectroscopy of Rubbers and Rubbery Materials, Rapra Technology Limited, Shropshire, UK, 2002, p. 265.

[32] J.-B. Donnet, R.C. Bansal, M.-J. Wang, Carbon Black: Science and Technology, Marcel Dekker Inc., New York, 1993, p. 155.

[33] ASTM D3192-09, Standard Test Methods for Carbon Black Evaluation in NR (Natural Rubber), ASTM International, West Conshohocken, PA, 2019.

[34] ASTM D4482-11, Standard Test Method for Rubber Property-Extension Cycling Fatigue, ASTM International, West Conshohocken, PA, 2017.

[35] J. Baum, A. Pines, Multiple-quantum NMR studies of clustering in solids, J. Am. Chem. Soc. 108 (1986) 7447-7454.

[36] K. Saalwächter, P. Ziegler, O. Spyckerelle, B. Haidar, A. Vidal, J.U. Sommer, ${ }^{1} \mathrm{H}$ multiple-quantum nuclear magnetic resonance investigations of molecular order distributions in poly (dimethylsiloxane) networks: evidence for a linear mixing law in bimodal systems, J. Chem. Phys. 119 (2003) 3468-3482.

[37] D.R. Burfield, K.L. Lim, Differential scanning calorimetry analysis of natural rubber and related polyisoprenes. Measurement of the glass transition temperature, Macromolecules 16 (1983) 1170-1175.
[38] W. Chassé, J.L. Valentín, G.D. Genesky, C. Cohen, K. Saalwächter, Precise dipolar coupling constant distribution analysis in proton multiple-quantum NMR of elastomers, J. Chem. Phys. 134 (2011) 044907.

[39] A.R. Payne, The dynamic properties of carbon black-loaded natural rubber vulcanizates. Part I, J. Appl. Polym. Sci. 6 (1962) 57-63.

[40] G. Kraus, Mechanical losses in carbon-black filled rubbers, J. Appl. Polym. Sci. Appl. Polym. Symp. 39 (1984) 75-92.

[41] S.M. Nagaraja, A. Mujtaba, M. Beiner, Quantification of different contributions to dissipation in elastomer nanoparticle composites, Polymer 111 (2017) 48-52.

[42] D. Merger, M. Abbasi, J. Merger, A.J. Giacomin, C. Saengow, M. Wilhelm, Simple scalar model and analysis for large amplitude oscillatory shear, Appl. Rheol. 5 (2016) 12-26.

[43] J.U. Sommer, W. Chassé, J.L. Valentín, K. Saalwächter, Effect of excluded volume on segmental orientation correlations in polymer chains, Phys. Rev. E 78 (2008) 051803.

[44] K. Saalwächter, Multiple-quantum NMR studies of anisotropic polymer chain dynamics, in: G. Webb (Ed.), Modern Magnetic Resonance, Springer, 2018, p. 755.

[45] C.R. Parks, O. Lorenz, Effect of network structure on aging of natural rubber vulcanizates, Ind. Eng. Chem. Prod. Res. Dev. 2 (1963) 279-284.

[46] J. Zhi, Q. Wang, M. Zhang, Z. Zhou, A. Liu, Y. Jia, Coupled analysis on hyperviscoelastic mechanical behavior and macromolecular network alteration of rubber during thermo-oxidative aging process, Polymer 171 (2019) 15-24.

[47] Y. Zhang, J.R. Choi, S.J. Park, Enhancing the heat and load transfer efficiency by optimizing the interface of hexagonal boron nitride/elastomer nanocomposites for thermal management applications, Polymer 143 (2018) 1-9.

[48] G. Kraus, J. Dugone, Adsorption of elastomers on carbon black, Ind. Eng. Chem. 47 (1955) 1809-1816.

[49] G. Kraus, J. Dugone, Adsorption of elastomers on carbon black, Rubber Chem. Technol. 29 (1956) 148-165.

[50] H.H. Le, S. Ilisch, H.J. Radusch, Characterization of the effect of the filler dispersion on the stress relaxation behavior of carbon black filled rubber composites, Polymer 50 (2009) 2294-2303.

[51] V. Hirschberg, L. Schwab, M. Cziep, M. Wilhelm, D. Rodrigue, Influence of molecular properties on the mechanical fatigue of polystyrene (PS) analyzed via Wöhler curves and Fourier Transform rheology, Polymer 138 (2018) 1-7.

[52] I.M. Ward, J. Sweeney, Mechanical Properties of Solid Polymers, John Wiley \& Sons, Chichester, UK, 2012, pp. 121-150. 
Karlsruher Institut für Technologie

\section{Repository KITopen}

Dies ist ein Postprint/begutachtetes Manuskript.

\section{Empfohlene Zitierung:}

Nie, S.; Lacayo-Pineda, J.; Willenbacher, N.; Wilhelm, M.

Aging of natural rubber studied via Fourier-transform rheology and double quantum NMR to correlate local chain dynamics with macroscopic mechanical response.

2019. Polymer, 181.

doi: $10.5445 / / R / 1000098519$

Zitierung der Originalveröffentlichung:

Nie, S.; Lacayo-Pineda, J.; Willenbacher, N.; Wilhelm, M.

Aging of natural rubber studied via Fourier-transform rheology and double quantum NMR to correlate local chain dynamics with macroscopic mechanical response. 2019. Polymer, 181, Article: 121804.

doi: 10.1016/j.polymer.2019.121804 